\title{
A new multi-dimensional flamelet generated manifolds approach for approximating partially premixed flame structure
}

\author{
Yuxin WU*, Changmin CAO*, Taohong YE* and Qizhao LIN* \\ *Department of Thermal Science and Energy Engineering, \\ University of Science and Technology of China \\ No.96, JinZhai Road Baohe District, Hefei, Anhui, 230026, China \\ E-mail: thye@ustc.edu.cn
}

Received 4 February 2015

\begin{abstract}
A new multi-dimensional flamelet generated manifolds(MFM) approach is proposed based on solving multi-dimensional flamelet equation set in mixture fraction $\mathrm{Z}$ and normalized flamelet progress variable $c$ coordinate, to capture both non-premixed and premixed combustion characteristics in partially premixed flames. Local scalar dissipation rates appeared as coefficients in multi-dimensional flamelet equation set have been modeled based on the analysis of local combustion regime during multi-dimensional flamelet calculation. Simulation results of counter-flow laminar partially premixed flames suggests that this new MFM approach can reproduce both non-premixed and premixed flame structure accurately, with computational efforts greatly reduced compared to former MFM approach.
\end{abstract}

Key words : Multi-dimensional flamelet generated manifolds, Laminar partially premixed flame, Combustion regime index

\section{Introduction}

Modeling of combustion systems often requires adequate dimension reduction procedures since detailed chemistry calculation of a number of chemical reactions will lead to prohibitive computational burden. For instance, intrinsic-low dimensional manifolds(ILDM) (Maas and Pope, 1992a, 1992b) and computational singular perturbation(CSP) (Lam, 1993) have been introduced into combustion simulation to reduce the computational costs. Further approaches based on ILDM like flame prolongation of ILDM(FPI) (Gicquel, et al., 2000) and flamelet generated manifolds(FGM) (Oijen and Goey, 2000) have also been developed in recent years. In the FGM approach, one-dimensional flamelet equations are solved to create a series of low-dimensional manifolds which could capture the main characteristic of flamefront. Via decoupling flow and chemical reactions, numerical computational costs can be drastically reduced.

FGM approach has been applied to both laminar and turbulent flame simulation successfully by using non-premixed or premixed generated manifolds respectively. (Peters, 1984, 1986. Pitsch, et al., 1995. Marracino and Lentini, 1997. Pitsch, 2002. Barths, et al., 1998. Coelho and Pitsch, 2001. Pierce, 2004. See and Ihme, 2015) When it comes to a flame controlled by partially premixed characteristic, existing models have certain drawbacks. For instance, non-premixed FGM cannot capture the propagation characteristic of partially premixed flame, while diffusive characteristic across iso-mixture fraction surfaces cannot be correctly captured by premixed FGM. To overcome such deficiencies, flamelet equation set in multi-dimensional composition space is deducted from the basic transport equation set of reacting flow by choosing specific flamelet coordinate. Thermo-chemical states obtained by solving multi-dimensional flamelet equations can be tabulated to form the multi-dimensional flamelet generated manifolds(MFM). (Nguyen, et al., 2010) For example, a multi-dimensional flamelet generated manifolds for partially premixed flame simulation can be obtained through designating mixture fraction $\mathrm{Z}$ and flamelet progress variable $Y_{c}$ as components of composition space coordinate. Consequently, partial premixed flame structure could be captured by solving both non-premixed and premixed contribution terms in multi-dimensional flamelet equation set to improve the modeling capability of existing FGM approach. 
As coefficients of multi-dimensional flamelet equations, scalar dissipation rates of mixture fraction $\mathrm{Z}$ and reaction progress variable $Y_{c}$ have to be modeled priori to solving the $Z-Y_{c}$ multi-dimensional flamelet equations. An asymptotic approximation distribution from counter-flow non-premixed flame could be used for modeling of scalar dissipation rate of mixture fraction $\chi_{Z}$ with strain rate $a$ introduced. (Peters, 1984, 2000) While for scalar dissipation rates of reaction progress variable $\chi_{Y_{c}}$, it's difficult to propose a generic distribution since gradient of $Y_{c}$ is influenced by both small scale mixing and chemistry. Nguyen proposed a modeling scheme for $\chi_{Y_{c}}$ by introducing a new flamelet library tabulation parameter $b_{s}$, distribution of $\chi_{Y_{c}}$ can be modeled as a functional form related to $b_{s}$ with a distribution form taken from laminar premixed flamelets. Since a multi-dimensional flamelet has to be calculated for each $a$ and $b_{s}$ value, computational cost is increased by two orders of magnitude compared to non-premixed and premixed flamelet models. Meanwhile, since the thermo-chemistry library is of four dimensions, which is too large to be implemented in combustion simulation especially for turbulent combustion modeling.

The motivation of the present research is to propose a new MFM approach to simplify the modeling scheme for scalar dissipation rates, in order to reduce the computational costs of flamelet tabulation. A normalized flamelet progress variable $c$ was introduced to redefine the coordinate system as Z-c in present paper, while an asymptotic approximation distribution for scalar dissipation rates $\chi_{c}$ and $\chi_{Z, c}$ can be modeled. A new multi-dimensional flamelet generated manifolds approach calculated in Z-c subspace coordinated has been developed, and simulations of a series of counter-flow laminar partially premixed flames have been carried out to verify the modeling capability of this new approach.

\section{Z-c multi-dimensional flamelet generated manifolds \\ 2.1 A multi-dimensional flamelet in Z-c coordinate}

To capture both non-premixed and premixed flame characteristic, a non-premixed flamelet with strain rate $a$ imposed propagating along progress variable direction, which forms a multi-dimensional flamelet, is considered in the present research. A normalized flamelet progress variable $c$ is introduced as $c=\frac{Y_{c}}{Y_{c}^{\max }(Z)}$ and multi-dimensional flamelet can be calculated in Z-c phase space. $c=0$ corresponds to frozen flow mixing, while $c=1$ represents an equilibrium status where $Y_{c}=Y_{c}^{\max }(Z)$ has reached its maximum value for fixed $\mathrm{Z}$. The calculated multi-dimensional flamelet can be illustrated as a flamelet with multi-regime flame structure consisting both non-premixed and premixed flame branches. Using flamelet generated transformation (Dold, 1989) while assuming unity Lewis number for $c$ and $Y_{c}$, transport equations of species mass fraction and temperature in $(Z, c)$ coordinate can be obtained,

$$
\begin{gathered}
\rho \frac{\partial Y_{i}}{\partial \tau}+\frac{\partial Y_{i}}{\partial c}\left[\rho \frac{\partial c}{\partial t}+\rho \mathbf{u} \cdot \nabla c-\nabla \cdot(\rho D \nabla c)\right]=\frac{\rho \chi_{Z}}{L e_{i}} \frac{\partial^{2} Y_{i}}{\partial Z^{2}}+\frac{\rho \chi_{c}}{L e_{i}} \frac{\partial^{2} Y_{i}}{\partial c^{2}}+2 \frac{\rho \chi_{Z, c}}{L e_{i}} \frac{\partial^{2} Y_{i}}{\partial Z \partial c}+\dot{\omega}_{i} \\
\rho \frac{\partial T}{\partial \tau}+\frac{\partial T}{\partial c}\left[\rho \frac{\partial c}{\partial t}+\rho \mathbf{u} \cdot \nabla c-\nabla \cdot(\rho D \nabla c)\right] \\
-\frac{1}{C_{p}}\left(\frac{\partial C_{p}}{\partial c}+\sum_{i=1}^{N} \frac{C_{p_{i}}}{L e_{i}} \frac{\partial Y_{i}}{\partial c}\right)\left(\frac{\partial T}{\partial c} \rho \chi_{c}+\frac{\partial T}{\partial Z} \rho \chi_{Z, c}\right)-\frac{1}{C_{p}}\left(\frac{\partial C_{p}}{\partial Z}+\sum_{i=1}^{N} \frac{C_{p_{i}}}{L e_{i}} \frac{\partial Y_{i}}{\partial Z}\right)\left(\frac{\partial T}{\partial Z} \rho \chi_{Z}+\frac{\partial T}{\partial c} \rho \chi_{Z, c}\right) \\
=\rho \chi_{Z} \frac{\partial^{2} T}{\partial Z^{2}}+\rho \chi_{c} \frac{\partial^{2} T}{\partial c^{2}}+2 \rho \chi_{Z, c} \frac{\partial^{2} T}{\partial Z \partial c}-\frac{1}{C_{p}} \sum_{i=1}^{N} h_{i} \dot{\omega}_{i}
\end{gathered}
$$

where $\chi_{Z}=\left(\lambda / \rho C_{p}\right)|\nabla Z|^{2}, \chi_{c}=\left(\lambda / \rho C_{p}\right)|\nabla c|^{2}, \chi_{Z, c}=\frac{\lambda}{\rho C_{p}} \nabla Z \cdot \nabla c=\mathbf{n}_{Z} \cdot \mathbf{n}_{c}\left(\chi_{Z} \chi_{c}\right)^{1 / 2} . \mathbf{u}$ is the velocity vector. $\chi_{c}$ represents scalar dissipation rate of normalized flamelet progress variable $c$, while $\chi_{Z, c}$ is the cross dissipation rate of $\mathrm{Z}$ and $c$. Normal unit vectors of iso- $Z$ and iso-c surfaces are defined by $\mathbf{n}_{Z}=-\nabla Z /|\nabla Z|$ and $\mathbf{n}_{c}=-\nabla c /|\nabla c| . \rho$ is mixture density. $C_{p_{i}}, D_{i}, h_{i}$, and $\dot{\omega}_{i}$ represents specific heat capacity, diffusive coefficient, specific enthalpy and reaction rate of the ith species respectively, while $L e_{i}=\left(\lambda / \rho C_{p}\right) / D_{i}$ represents the Lewis number of the ith species. $\lambda$ 
and $C_{p}$ each stands for heat transfer and specific heat coefficient of the mixture.

Equations (1) and (2) constitute the multi-dimensional flamelet equation set. The term $\left[\rho \frac{\partial c}{\partial t}+\rho \mathbf{u} \cdot \nabla c-\nabla \cdot(\rho D \nabla c)\right]$ is considered as a source term of $c$, which can be closed by setting $Y_{i}=Y_{c}$ in Eq. (1),

$$
\rho \frac{\partial c}{\partial t}+\rho \mathbf{u} \cdot \nabla c-\nabla \cdot(\rho D \nabla c)=\left(\frac{\partial Y_{c}}{\partial c}\right)^{-1}\left(-\rho \frac{\partial Y_{c}}{\partial \tau}+\rho \chi_{Z} \frac{\partial^{2} Y_{c}}{\partial Z^{2}}+\rho \chi_{c} \frac{\partial^{2} Y_{c}}{\partial c^{2}}+2 \rho \chi_{Z, c} \frac{\partial^{2} Y_{c}}{\partial Z \partial c}+\dot{\omega}_{Y_{c}}\right),
$$

Assuming flame front of the studied multi-dimensional flamelet is located at $c=c^{*}(Z)$ where highest reaction rates has reached, preheat region is found for $c<c^{*}(Z)$ and left burnt gas is found for $c>c^{*}(Z)$. As for regions with $c$ value close to unity, convection and diffusion terms along $c$ direction vanished since $c$ taken a constant unity value, which leads to a diffusive flame branch. Under the lower strain rate, multi-dimensional flamelet is weakly influenced by diffusive fluxes across iso-Z surfaces, and propagates in a premixed flamelet way. Increasing strain rate will enhance diffusive contribution leading to a partially premixed flame structure as in strained triple flame. (Ghosal and Vervisch, 2000. Domingo, et al., 2005. Pitsch and Duchamp, 2002) Further increasing strain rate will lead to a trailing diffusion flame. Both non-premixed and premixed flame structures can be found during MFM calculation, as in a strained partially premixed flame.

\subsection{Modeling of scalar dissipation rates}

To solve the multi-dimensional flamelet equations (1) and (2) in Z-c coordinate, coefficients such as scalar dissipation rates $\chi_{Z}, \chi_{c}$ and $\chi_{Z, c}$ need to be modeled. Mixture fraction dissipation $\chi_{Z}$ can be assumed to have an asymptotic approximation distribution as in counter-flow non-premixed flame since it is mainly influenced by flow mixing[14],

$$
\chi_{Z}=\frac{a}{2 \pi} \exp \left[-2\left(\operatorname{erfc}^{-1}(2 Z)\right)^{2}\right]=\chi_{s t} \exp \left[-2\left(\operatorname{erfc}^{-1}(2 Z)\right)^{2}\right] / \exp \left[-2\left(\operatorname{erfc}^{-1}\left(2 Z_{s t}\right)\right)^{2}\right]
$$

where $\chi_{s t}$ represents the stoichiometric scalar dissipation rate of mixture fraction under each strain rate $a$. Since scalar dissipation rates $\chi_{c}$ and $\chi_{Z, c}$ are related to gradient $\nabla c$, which need to be evaluated. The distribution of $\nabla c$ value has been investigated for the calculated multi-dimensional flamelet in the following context.

\subsubsection{Estimation of $\nabla c$ value distribution}

In order to predict the value of $\nabla c$, Eq. (3) need to be obtained by $Y_{i}=Y_{c}$ in flamelet equation (1). Term $\frac{\partial Y_{c}}{\partial \tau}$ related to time $\tau$ describing the evolution of flamelet can be neglected after solutions have reached convergence. Meanwhile burning velocity $S_{L, u}$ could be introduced to separate steady and unsteady phenomena of Eq. (3), leading to

$$
\begin{gathered}
\frac{\partial Y_{c}}{\partial c}\left(\rho \frac{\partial c}{\partial t}+\left(\rho \mathbf{u}-\rho_{u} s_{L, u} \mathbf{n}\right) \cdot \nabla c\right)_{1}+\left(\frac{\partial Y_{c}}{\partial c}\left[\rho_{u} s_{L, u}|\nabla c|-\nabla \cdot(\rho D \nabla c)\right]-\rho \chi_{c} \frac{\partial^{2} Y_{c}}{\partial c^{2}}\right)_{2} \\
+\left(-\rho \chi_{Z} \frac{\partial^{2} Y_{c}}{\partial Z^{2}}\right)_{3}+\left(-2 \rho \chi_{Z, c} \frac{\partial^{2} Y_{c}}{\partial Z \partial c}\right)_{4}=\dot{\omega}_{Y_{c}}
\end{gathered}
$$

where $\rho_{u}$ represents the unburnt density as in premixed flamelet, $\mathbf{n}=\nabla c /|\nabla c|$ is the normal vector representing the direction of the gradient of $c$. Group 1 terms on the left describe the propagating iso- $c$ surface as in G-equation(Domingo, et al., 2002) and can be assumed to be neglected under steady assumption in the present research. Group 2 terms represent convection and diffusion contributions along $c$ direction. Group 3 terms represent diffusive fluxes across iso-Z surfaces, and group 4 terms correspond to cross diffusion contributions.

To achieve high modeling accuracy, analysis of local gradient $\nabla c$ in partially premixed flame has to be performed during multi-dimensional flamelet calculation, especially for regions with high reaction rates where present diffusion flame or premix flame regime respectively.

Firstly, in flame region piloted mainly by premixed flame regime, where $\chi_{Z}$ is relatively small and local $\dot{\omega}_{Y_{c}}$ is of a high value, group 3 terms are relatively small compared to group 2 terms. Since transport between fresh and burnt gases at 
flame front is one-dimensional along -n direction and transport contributions along other directions can be neglected. (Knudsen and Pitsch, 2009) $\chi_{Z, c}=0$ can then be assumed and group 4 terms in Eq.(5) vanish. Based on above analysis, local reaction rates are mainly balanced by group 2 terms as in a premixed flamelet, leading to the suggestion that local species" gradients are driven by local premixed flame structure. Gradient $\nabla Y_{c}$ value is assumed to have the relation as $\nabla Y_{c}\left(Z, Y_{c}\right)=\nabla Y_{c}^{\text {pre }}\left(Z, Y_{c}\right)$, with $\nabla Y_{c}^{\text {pre }}\left(Z, Y_{c}\right)$ pre-tabulated using single-dimensional unstrained premixed FGM approach. Gradient $\nabla c$ value can be deducted using this relation as $\nabla c\left(Z, Y_{c}\right)=\nabla c^{p r e}\left(Z, Y_{c}\right) \frac{Y_{c}^{e q}(Z)}{Y_{c}^{\max }(Z)}$, yielding

$$
\chi_{c}\left(Z, Y_{c}\right)=\chi_{c}^{\text {pre }}\left(Z, Y_{c}\right)\left(\frac{Y_{c}^{e q}(Z)}{Y_{c}^{\max }(Z)}\right)^{2}
$$

where $Y_{c}^{e q}(Z)$ represents the equilibrium value of progress variable $Y_{c}$ in premixed FGM at a given mixture franction $\mathrm{Z}$.

Secondly, in flame region dominated by diffusion regime where an equiblirum status has reached and $Y_{c}$ is taken its maximum value $Y_{c}^{\max }(Z)$ for fixed mixture fraction $\mathrm{Z}$, an evenly distributed $c$ having the constant value $c=1$ could be assumed and the values of $\nabla c, \chi_{c}$ and $\chi_{Z, c}$ will tend to zero. Group 2 terms and term 4 in Eq. (5) will vanish leading to the balance between local reaction rate and group terms 3 only, which is identical to non-premixed flamelet.

Since local gradient of normalized progress variable value $\nabla c$ can be predicted in premixed or non-premixed branch by different modeling schemes respectively, a linear assumption could made to approximate its distribution in the rest of partially premixed flame, based on determination of local flame structure using a criterion approach in the following context.

\subsubsection{Modeling of $\chi_{c}$ and $\chi_{Z, c}$}

To propose an accurate approximate distribution for the gradient $\nabla c$, local flame structure has to be related with certain criterion. An index $\xi$ is proposed as Eq.(7), taking a form similar to the combustion index concept proposed by Knudsen et.al., (Knudsen and Pitsch, 2012)

$$
\xi=\frac{\frac{\partial Y_{c}}{\partial c}\left[\rho_{u} s_{L, u}|\nabla c|-\nabla \cdot(\rho D \nabla c)\right]-\rho \chi_{c} \frac{\partial^{2} Y_{c}}{\partial c^{2}}}{\dot{\omega}_{Y_{c}}}=\frac{\dot{\omega}_{Y_{c}}+\rho \chi_{Z} \frac{\partial^{2} Y_{c}}{\partial Z^{2}}}{\dot{\omega}_{Y_{c}}}
$$

This new defined index $\xi$ represents how local reaction rate of flamelet progress variable $Y_{c}$ is balanced by different flame regimes. $\xi=0$ stands for diffusive flame branches where reaction rates are balanced by diffusion fluxes yielding $\dot{\omega}_{Y_{c}}=-\rho \chi_{Z} \frac{\mathrm{d}^{2} Y_{c}}{\mathrm{~d} Z^{2}} \quad$, while $\quad \xi=1$ for premixed flame branches where we have, $\dot{\omega}_{Y_{c}}=\frac{\partial Y_{c}}{\partial c}\left[\rho_{u} s_{L, u}|\nabla c|-\nabla \cdot(\rho D \nabla c)\right]-\rho \chi_{c} \frac{\partial^{2} Y_{c}}{\partial c^{2}}$, which is the same as in premixed flamelet without fluxes across iso-Z surfaces.

Since value of $\nabla c$ can be predicted at $\xi=0$ or $\xi=1$, a linear assumption is made to approximate its distribution according to $\xi$ value as in a very first approach, which will also leads to assumption for $\chi_{c}$ value as

$$
\chi_{c}\left(Z, Y_{c}\right)=\left[\nabla c\left(Z, Y_{c}\right)\right]^{2}=\xi^{2} \chi_{c}^{\text {pre }}\left(Z Y_{c}\right)\left(\frac{Y_{c}^{e q}(Z)}{Y_{c}^{\mathrm{ma}}(Z)}\right)^{2}
$$

As former discussed, $\chi_{c}^{\text {pre }}\left(Z, Y_{c}\right)$ and $Y_{c}^{e q}(Z)$ can be obtained from pre-calculated premixed FGM flamelet library. For cross dissipation rate $\chi_{Z, c}$, since its value is close to zero in both premixed and non-premixed flame regions as former discussed, assumption as $\chi_{Z, c}=0$ has been made for $0 \leq \xi \leq 1$.

All the above assumptions will prevail for flame regions governed mainly by single non-premixed or premixed regime, since $\xi \rightarrow 0$ corresponding to $\chi_{c} \rightarrow 0$ and $\chi_{Z, c} \rightarrow 0$, while $\xi \rightarrow 1$ leads to $\chi_{c}\left(Z, Y_{c}\right) \rightarrow \chi_{c}^{p r e}\left(Z, Y_{c}\right)\left(\frac{Y_{c}^{e q}}{Y_{c}^{\max }}\right)^{2}$ and 
$\chi_{Z, c} \rightarrow 0$. Based on the above analysis, equations (7) and (8) can be coupled to provide an implicit relation between the value of $\chi_{c}$ and local multi-dimensional flamelet structure, which will make the solution of MFM equation set feasible with $\chi_{c}^{\text {pre }}\left(Z, Y_{c}\right)$ and $Y_{c}^{e q}(Z)$ values pre-tabulated from premixed FGM approach.

\subsection{Z-c multi-dimensional flamelet tabulation}

Based on the former discussions, partially premixed flamelet library can be tabulated by calculating a series of Z-c multi-dimensional flamelets with different stoichiometric scalar dissipation rate $\chi_{s t}$ corresponding to different strain rate $a$ values imposed. Scalar dissipation rate $\chi_{Z}$ is assumed to have a distribution as Eq. (4), while distributions of $\chi_{c}$ and $\chi_{Z, c}$ values can be modeled during calculation as former discussed in section 2.2.

Fixed value boundary conditions are imposed during flamelet calculation by setting oxidizer stream with $Z=0$, fuel stream with $Z=1$ and frozen mixing stream with $c=0$. For diffusive flame branch boundary where $c=1$, $\chi_{Z}=\chi_{Z, c}=0$ could be assumed, leading to the boundary condition $\dot{\omega}_{i}=-\rho \chi_{Z} \frac{\mathrm{d}^{2} Y_{c}}{\mathrm{dZ}^{2}}$ as in non-premixed flamelet.

After multi-dimensional flamelet calculation, using relation $Y_{c}=c \cdot Y_{c}^{\max }(Z)$ and the inverse form of Eq.(4), local species mass fractions and temperature of Z-c multi-dimensional flamelet library can be tabulated as

$$
Y_{i}=Y_{i}^{M F M}\left(Z, Y_{c}, \chi_{Z}\right), T=T^{M F M}\left(Z, Y_{c}, \chi_{Z}\right)
$$

taking a form as three-dimensional manifolds, with respect to local mixture fraction $\mathrm{Z}$, flamelet progress variable $Y_{c}$ and scalar dissipation rate $\chi_{z}$.

\subsection{Numerical algorism}

Since the governing equation set of MFM is of strong stiffness, strang splitting algorism (Strang, 1968) has been applied to decouple chemical and diffusive terms. Convection terms are predicted by AB2 scheme at each time step, while 8th Runge-Kutta-Chebychev integration (Verwer and Sommeijer, 2004) for diffusion terms is used. Reaction source terms are integrated using VODE solver. (Brown, et al., 2005)

\section{Z-c MFM flamelet tabulation for approximating partially premixed flame structure}

Counter-flow partially premixed flame simulation has been performed by Fiorina et.al to estimate the extension of premixed FPI into laminar partially premixed flames. (Fiorina, et al., 2005) Simulation results indicated that, close agreement has been found for FPI solutions with detailed chemistry ones for fuel stream equivalence ratio below rich flammability limit. However, this conclusion failed for flame with fuel stream equivalence ratio $\phi_{F}$ above rich flammability limit, since diffusive became non-negligible and cannot be captured by premixed flamelets.

To overcome the limitation of premixed FPI and FGM approaches, Z-c multi-dimensional flamelet library has been pre-tabulated to approximate both non-premixed and premixed flame structure of laminar partially premixed flames in present paper.

\subsection{Z-c MFM flamelet library tabulation}

Z-c multi-dimensional flamelet library has been built for methane-air combustion, with flamelet progress variable $Y_{c}$ defined as $Y_{c}=Y_{C O}+Y_{\mathrm{CO}_{2}}$. (Fiorina, et al., 2003. Fiorina, et al., 2005) Mixture fraction $Z=0$ corresponds to pure air stream, while $Z=1$ represents pure methane stream. $Z_{s t}=0.055$ corresponds to the mixture fraction value at stoichiometric equivalence ratio. Detailed boundary conditions are listed in Table 1. 
Table 1 Boundary conditions for multi-dimensional flamelet calculation.

\begin{tabular}{ccccc}
\hline & $Y_{\mathrm{CH}_{4}}$ & $Y_{\mathrm{O}_{2}}$ & $Y_{N_{2}}$ & $T$ \\
\hline$Z=0$ & 0.0 & 0.232 & 0.768 & $298 \mathrm{~K}$ \\
\hline$Z=1$ & 1.0 & 0.0 & 0.0 & $298 \mathrm{~K}$ \\
\hline$c=0$ & $Z$ & $0.232 \cdot(1-Z)$ & $0.768 \cdot(1-Z)$ & $298 \mathrm{~K}$ \\
\hline$c=1$ & \multicolumn{5}{c}{$\dot{\omega}_{i}=-\rho \chi_{Z} \frac{\mathrm{d}^{2} Y_{i}}{\mathrm{dZ}^{2}}$} \\
\hline
\end{tabular}

Reduced mechanism DRM-19 (Kazakov and Frenklach) based on GRI1.2 including 21 species has been used for chemistry solving. A mesh of size 200 and 100 in $\mathrm{Z}$ and $c$ directions respectively was used.

A series of multi-dimensional flamelets have been calculated in Z-c coordinate, assuming unity Lewis numbers for all species, with different stoichiometric scalar dissipation rate $\chi_{s t}$ corresponding to different strain rate $a$ imposed.

$\chi_{s t}=0 \mathrm{~s}^{-1}$ corresponds to premixed flamelet generated manifolds, while all the other non-zero $\chi_{s t}$ values lead to strained partially premixed flames. $\dot{\omega}_{Y_{c}}$ as the reaction rate of $Y_{c}$ can be plotted using a function form of Z-c for each $\chi_{s t}$ value. Fig. 1 shows the contour of reaction rate $\dot{\omega}_{Y_{c}}$ at $\chi_{s t}=1 \mathrm{~s}^{-1}$ in Z-c flamelet coordinate. Intense reacting region is found around $Z=Z_{s t}$ in mixture fraction space and $\dot{\omega}_{Y_{c}}$ value reaching its peak at flamefront $c=c^{*}(Z)$.

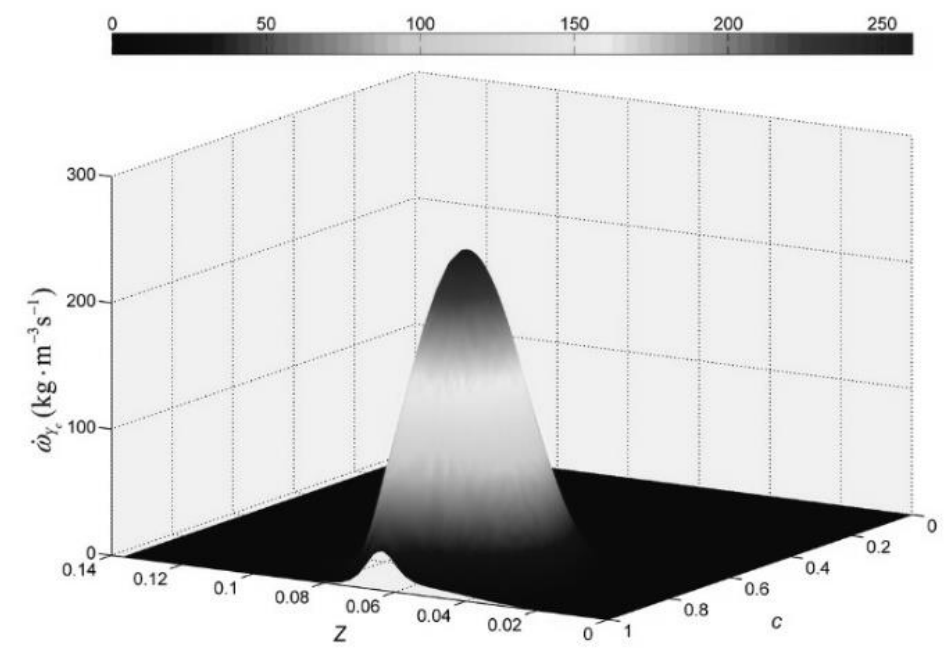

Fig. 1 Contour of reaction rate of $Y_{c}$ for $\chi_{s t}=1 \mathrm{~s}^{-1}$.

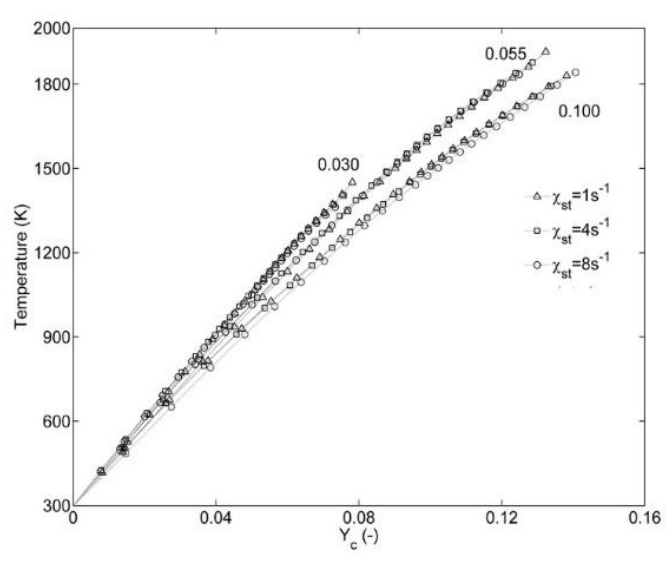

(a)

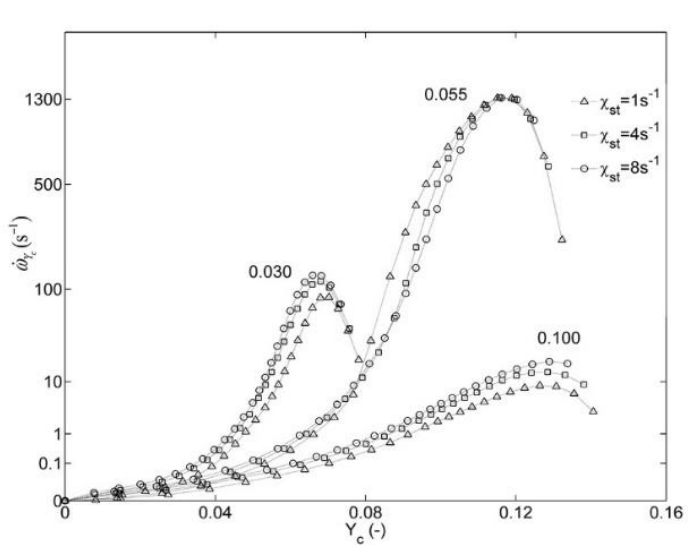

(b) 
Fig. 2 Flamelet trajectories in $Z-Y_{c}$ coordinate: (a) temperature (b) $\dot{\omega}_{Y_{c}}$. Delta: $\chi_{s t}=1 \mathrm{~s}^{-1}$; Circle: $\chi_{s t}=4 \mathrm{~s}^{-1}$, Square: $\chi_{s t}=8 \mathrm{~s}^{-1} . Z_{L}=0.030(\phi=0.53), Z_{s t}=0.055(\phi=1.0), Z_{R}=0.100(\phi=1.92)$.

In Fig. 2, Z-c MFM solutions at lean mixture branch with $Z_{L}=0.030$, stoichiometric mixture branch with $Z_{s l}=0.055$ and rich mixture branch with $Z_{R}=0.100$ are plotted in $Z-Y_{c}$ coordinate. Tendency of curves indicates that scalar dissipation rate of mixture fraction has an impact on the species distribution and the reaction rate of progress variable. Increasing the value of stoichiometric scalar dissipation rate $\chi_{s t}$ will lead to a thickened reacting zone in mixture fraction space, as the flame front spreading from stoichiometric position into lean and rich branch. Values of mass fraction $Y_{O H}$ and the reaction rate of progress variable $\dot{\omega}_{Y_{c}}$ decreases with the increase of $\chi_{s t}$ value in intense reaction region. While for lean and rich branch, a contrary tendency is observed.

\subsection{Counter-flow laminar partially premixed flame configurations}

Counter-flow flame (Li and Williams, 1999. Ravikrishna and Laurendeau, 2000. Barlow, et al., 2001) configuration is used to verify Z-c MFM modeling capability for laminar partially premixed methane-air flame simulation, by setting fuel inlet stream as mixture of methane and air.

Figure. 3 shows a counter-flow flame of axisymmetric configurations, using $\mathrm{x}$ to denote the radial coordinate, and $\mathrm{y}$ the axial one. $y \rightarrow-\infty$ represents the oxidizer stream with pure air(equivalence ratio $\phi=0$ ) and $y \rightarrow+\infty$ the fuel stream with methane and air mixture $\left(\phi=\phi_{F}\right) . \quad y=0$ corresponds to the stagnation space where velocity component along y direction reaches zero.

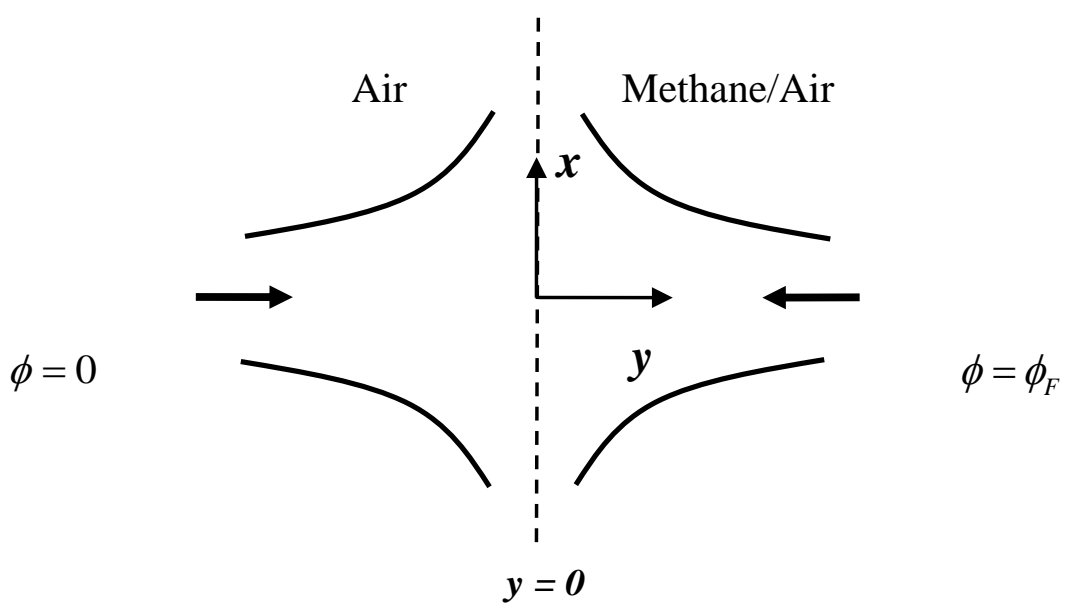

Fig. 3 Sketch of a counter-flow flame configuration. The dashed line represents the stagnation plane.

Strain rate $a$ for each counter-flow flame is measured by the velocity gradient away from the intense reaction zone. Detailed chemistry solutions for each flame are calculated using one-dimensional equation set, (Darabiha, 1992) with boundary conditions as

$$
v_{y=0}=0, \hat{u}_{y \rightarrow-\infty}=\left(\rho_{+\infty} / \rho_{-\infty}\right)^{0.5}, \hat{u}_{y \rightarrow+\infty}=1, \varphi_{y \rightarrow-\infty}=\varphi_{O}, \varphi_{y \rightarrow+\infty}=\varphi_{F} .
$$

where $j$ is set to be 1 in the case of axisymmetric configurations, $\hat{u}=u / a x$ is the reduced radial velocity. $\varphi$ denotes species mass fraction and temperature and $\varphi_{O}$ represents species mass fraction and temperature of oxidizer stream and $\varphi_{F}$ the fuel ones.

\subsection{Comparison among premixed FGM, Z-c MFM and detailed chemistry solutions}


To verify the modeling accuracy for this new proposed Z-c MFM approach, a posteriori verification has been carried out by comparing both premixed FGM and the present Z-c MFM solutions with detailed chemistry results.

DRM19 (Kazakov and Frenklach) mechanism including 21 species is used for describing chemistry reaction kinetics. A computer with an $17-2600 \mathrm{~K}$ processor is used during flamelet calculations. A series of premixed flamelets are calculated with CPU time of $0.5 \mathrm{~h}$, of which solutions are interpolated into a premixed FGM flamelet library $\left(Z, Y_{c}\right)$ of the size $(100 \times 100)$. Meanwhile a series of multi-dimensional flamelets with stoichiometric scalar dissipation rate $\chi_{s t}=0.1,0.2,0.4,1,2,4,6,8,10 \mathrm{~s}^{-1}$ are calculated with total CPU time of 5.4h, of which solutions are interpolated into a Z-c MFM flamelet library $\left(Z, Y_{c}, \chi_{Z}\right)$ of the size $(100 \times 100 \times 20)$. A mesh with 100 grids is applied during detailed chemistry calculations of counter-flow flames.

After detailed chemistry calculations of laminar counter-flow flames, premixed FGM solutions $\varphi^{F G M}\left(Z, Y_{c}\right)$ can be reproduced using local $\mathrm{Z}$ and $Y_{c}$ values of detailed chemistry solutions to look up premixed FGM flamelet library, while Z-c MFM ones $\varphi^{M F M}\left(Z, Y_{c}, \chi_{Z}\right)$ using local $Z, Y_{c}$ and scalar dissipation rate $\chi_{Z}$ values to look up Z-c MFM flamelet library. Major species' mass fraction and temperature values along flamelet trajectories have been plotted for each approach in following sections. Different flame regimes can be achieved by varying the ratio of inlet fuel premixed with air.

\subsubsection{Premixed flame against air}

For a premixed flame against air with stoichiometric mixture in fuel jet, a premixed flame burning layer is stabilized between fresh mixture and the stagnation plane. Comparisons among premixed FGM, Z-c MFM and detailed chemistry reference flames for temperature, mass fractions of $\mathrm{CO}_{2}$ and $\mathrm{OH}$, and reaction rate of progress variable $\dot{\omega}_{Y_{c}}$ under different strain rate $a$ are plotted along mixture fraction $\mathrm{Z}$ in Fig. 4.
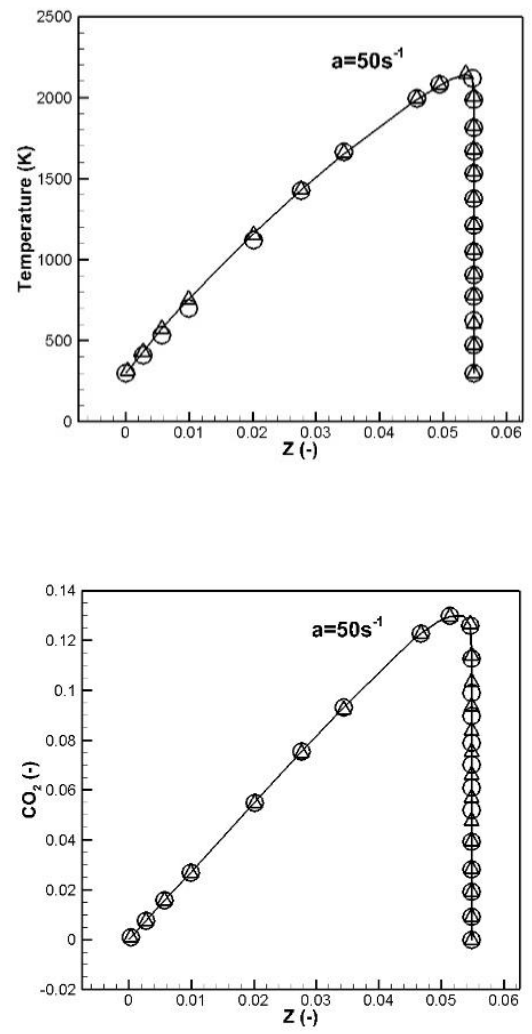

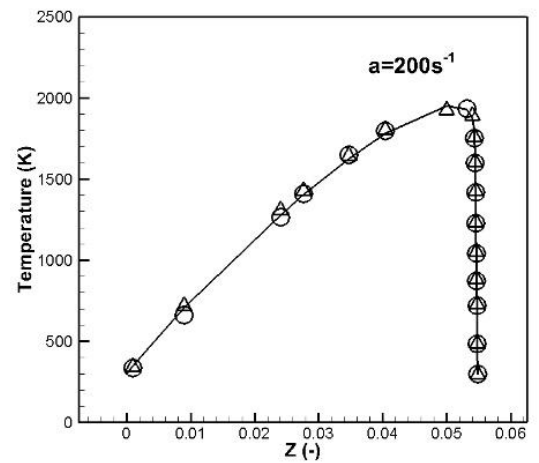

(a)

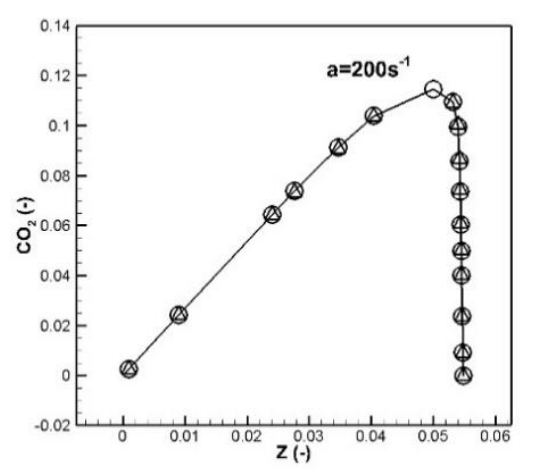

(b)
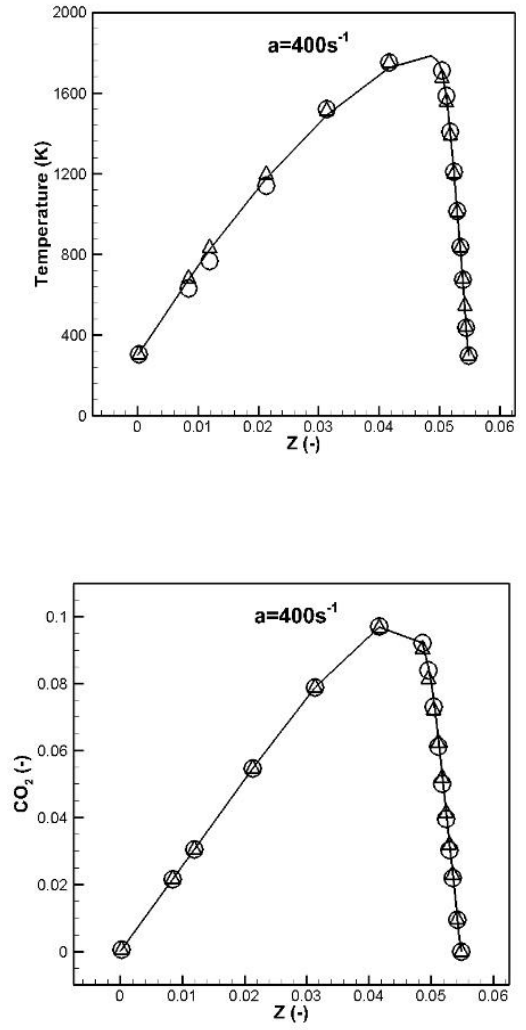

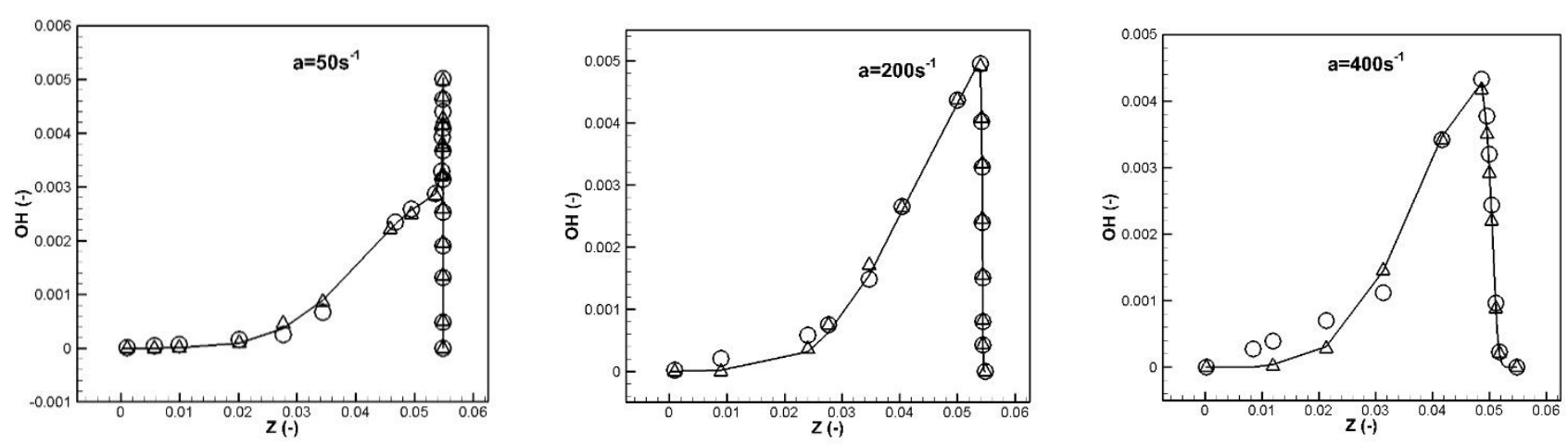

(c)
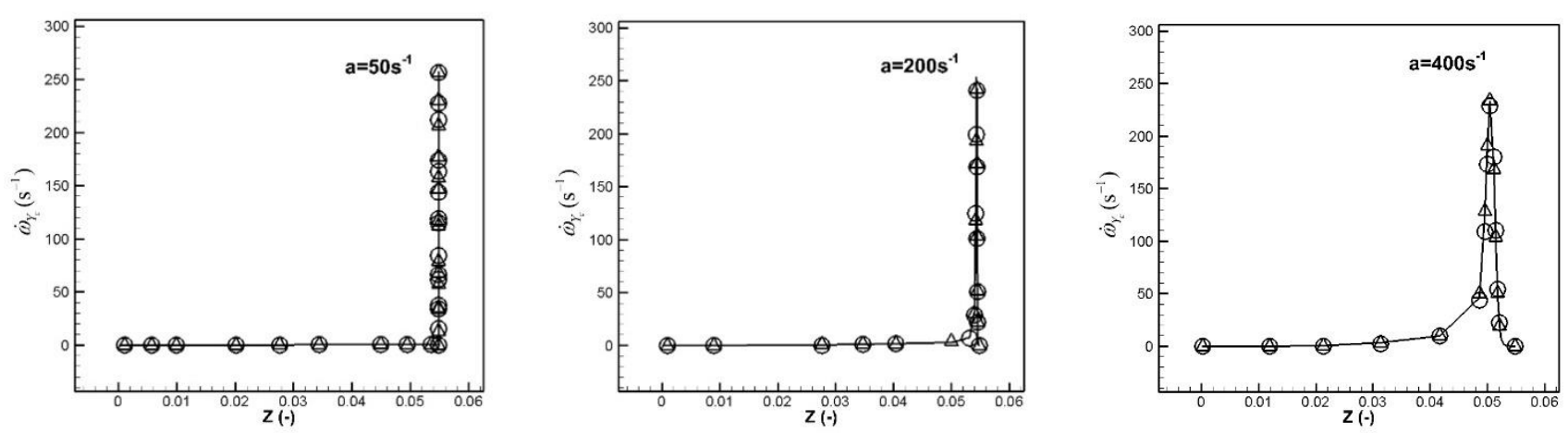

(d)

Fig. 4 Comparisons among different modeling solutions for flames with fuel equivalence ratio $\phi_{F}=1.0$ under different strain rate $a$. (a)Temperature, (b) $Y_{\mathrm{CO}_{2}}$, (c) $Y_{\mathrm{OH}}$, (d) $\dot{\omega}_{Y_{c}}$. Line: detailed chemistry reference solutions. Symbols: Circle, FGM solutions; Delta, Z-c MFM solutions.

Under the low strain rate $a=50 \mathrm{~s}^{-1}$ imposed at the counter-flow flame, a steep increase of temperature is observed around $Z_{s t}=0.055$ with intense reaction. A spreading of reaction zone into lower mixture fraction region is observed with the increase of strain rate $a$, since interactions between reaction layers at different equivalence ratios are enhanced. Results of premixed FGM and Z-c MFM both achieve good agreements with detailed chemistry solutions for calculated counter-flow flames with low strain rates imposed. For higher strain rate value as $a=400 \mathrm{~s}^{-1}$, premixed FGM approach can still reproduce local temperature $\mathrm{T}$, mass fraction of major specie $\mathrm{CO}_{2}$ and $\dot{\omega}_{Y_{c}}$ accurately. For intermediate specie $\mathrm{OH}$ at lean flame branch away from stoichiometric intense burning region, since diffusive fluxes cannot be neglected compared to local reaction rates, while Z-c MFM approach still proves to be accurate.

\subsubsection{Rich partially premixed flames}

For rich partially premixed flames against air with fuel equivalence ratio $\phi_{F}=1.6$ inside the flammability domain, comparisons among different modeling solutions are presented in Fig.5. 

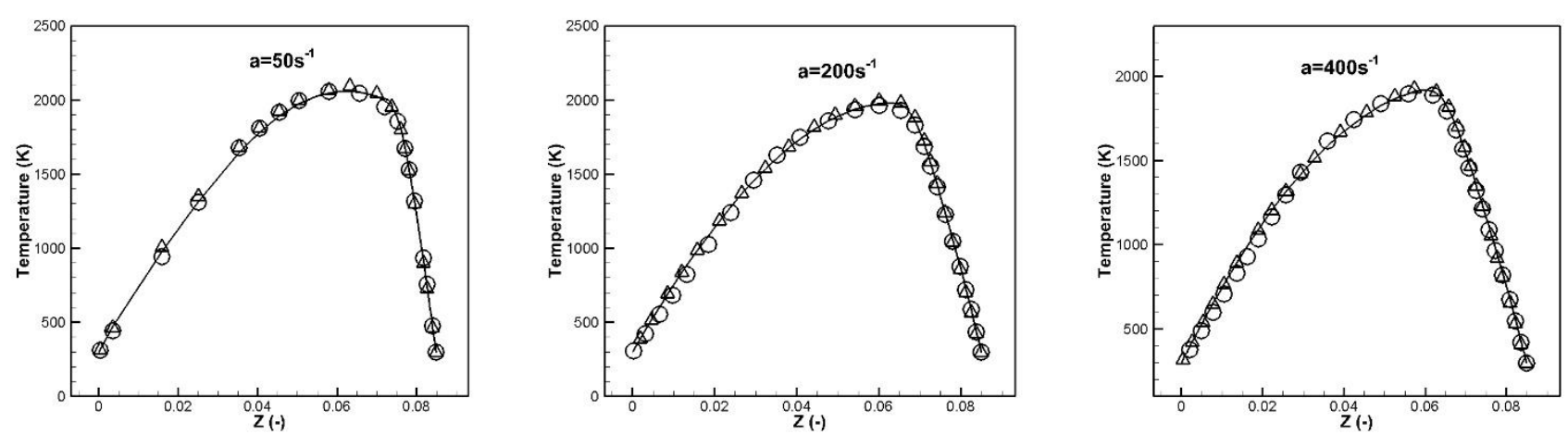

(a)
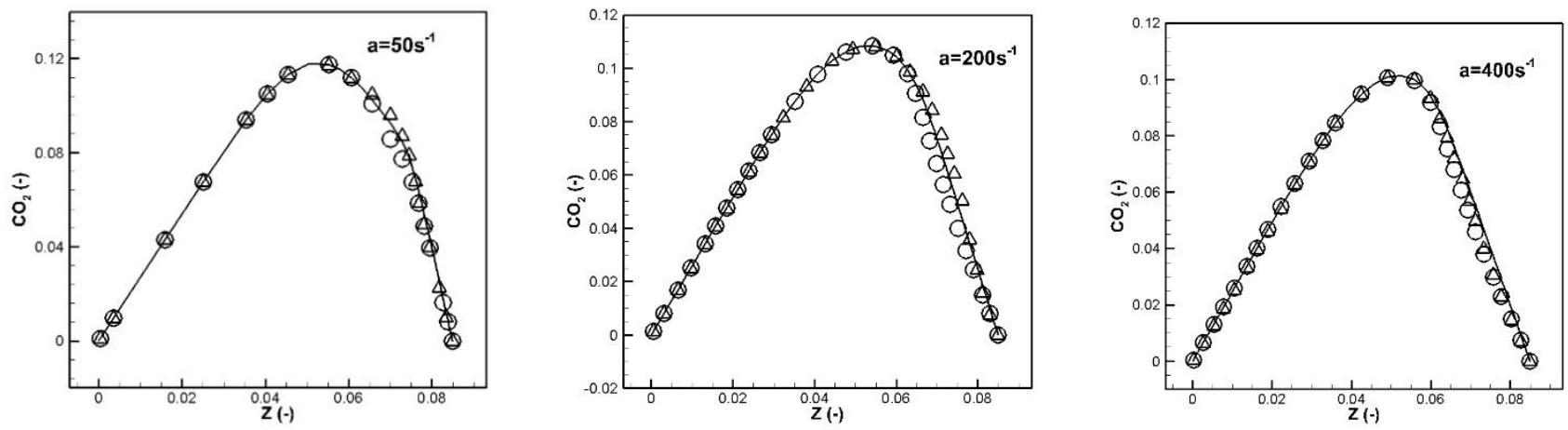

(b)
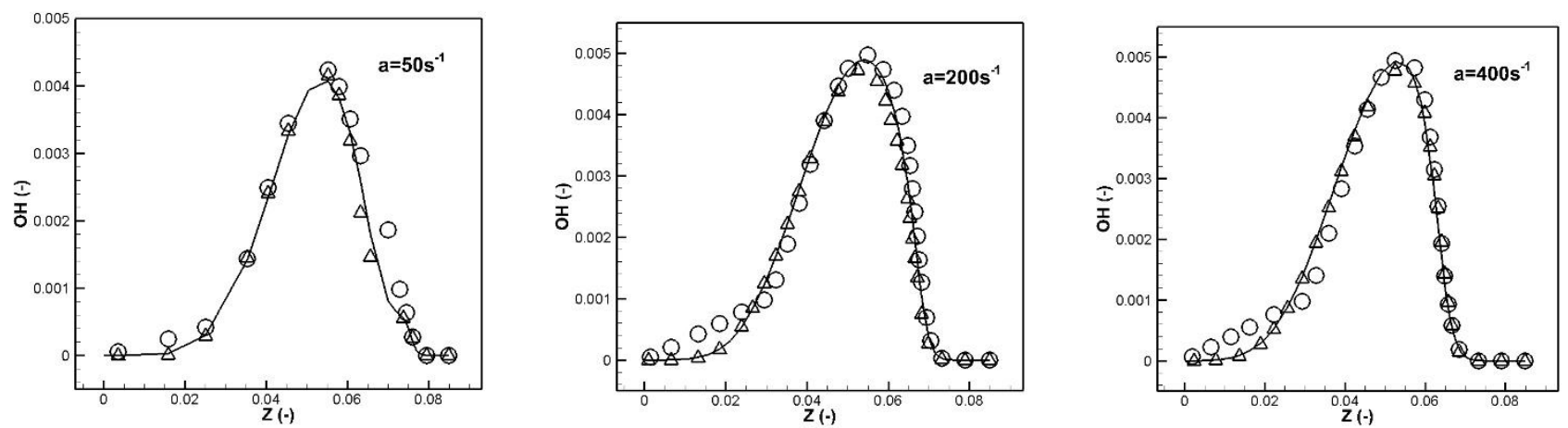

(c)
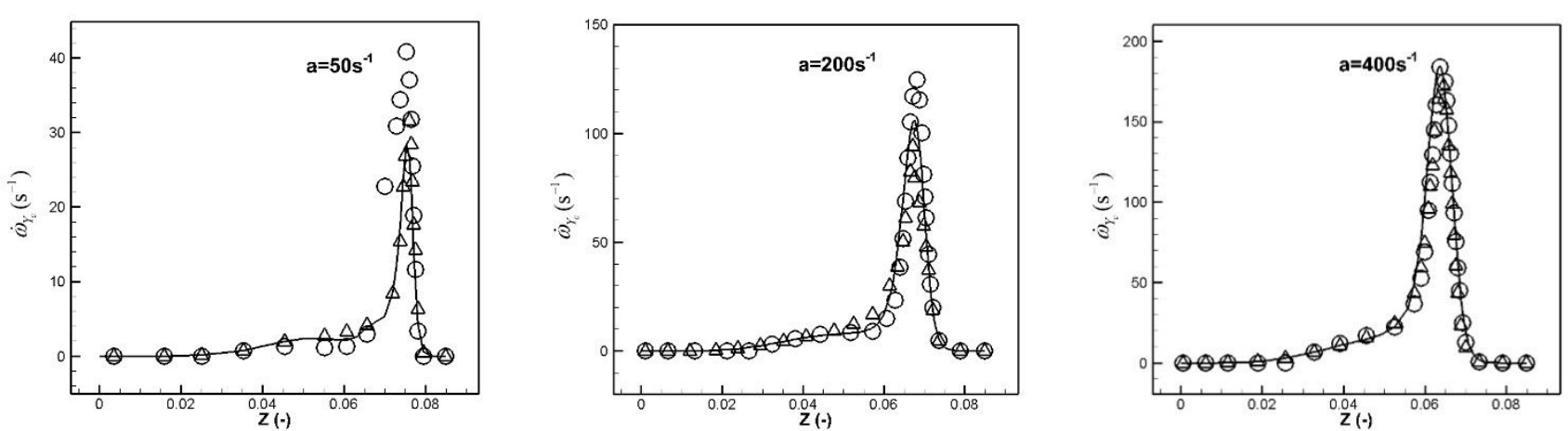

(d) 
Fig. 5 Comparisons among different modeling solutions for flames with fuel equivalence ratio $\phi_{F}=1.6$ under different strain rate $a$. (a)Temperature, (b) $Y_{\mathrm{CO}_{2}}$, (c) $Y_{\mathrm{OH}}$, (d) $\dot{\omega}_{Y_{c}}$. Line: detailed chemistry reference solutions. Symbols: Circle, FGM solutions; Delta, Z-c MFM solutions.

Temperature and mass fraction of $\mathrm{CO}_{2}$ are well predicted by both premixed FGM and Z-c MFM approach. However, for mass fraction of intermediate specie $\mathrm{OH}$, Z-c MFM approach can still reproduce its distribution accurately while premixed FGM failed. Both premixed FGM and Z-c MFM solutions cannot provide an accurate prediction for $\dot{\omega}_{Y_{c}}$ close to detailed chemistry solutions, since interactions between diffusive and premixed layer are enhanced in rich laminar partially premixed flames, while the linear modeling distributions of scalar dissipation rates $\chi_{c}$ and $\chi_{Z, c}$ lose its accuracy for regions where $\xi$ value is not close to zero or unity in Z-c MFM approach.

For rich partially premixed flames against air with fuel equivalence ratio $\phi_{F}=5.0$ outside the flammability domain governed by diffusion regime mainly, comparisons among different modeling solutions are presented in Fig.6.
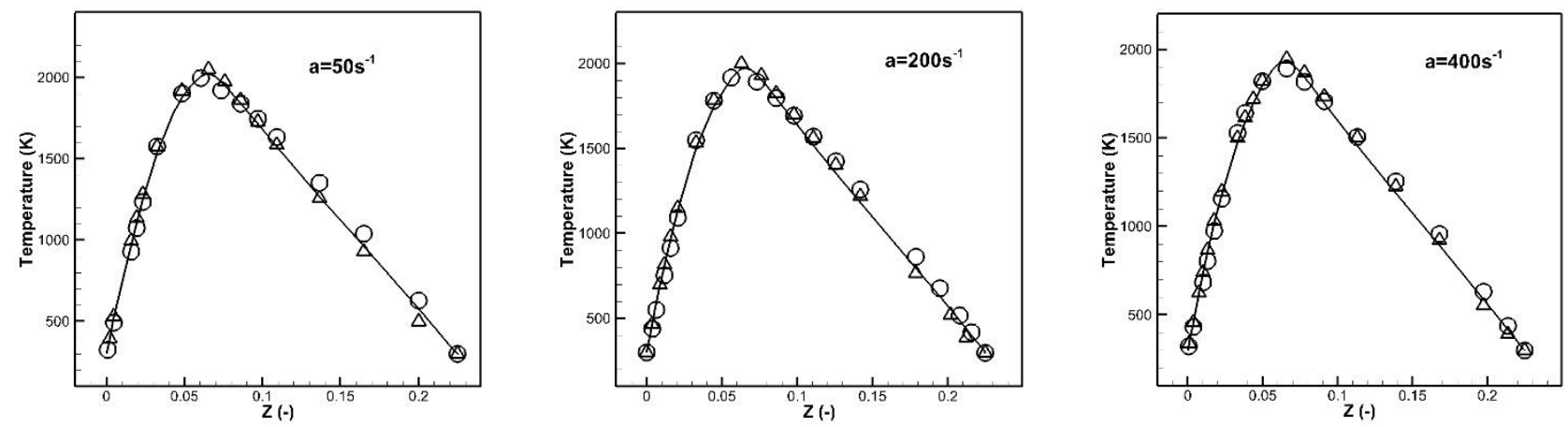

(a)
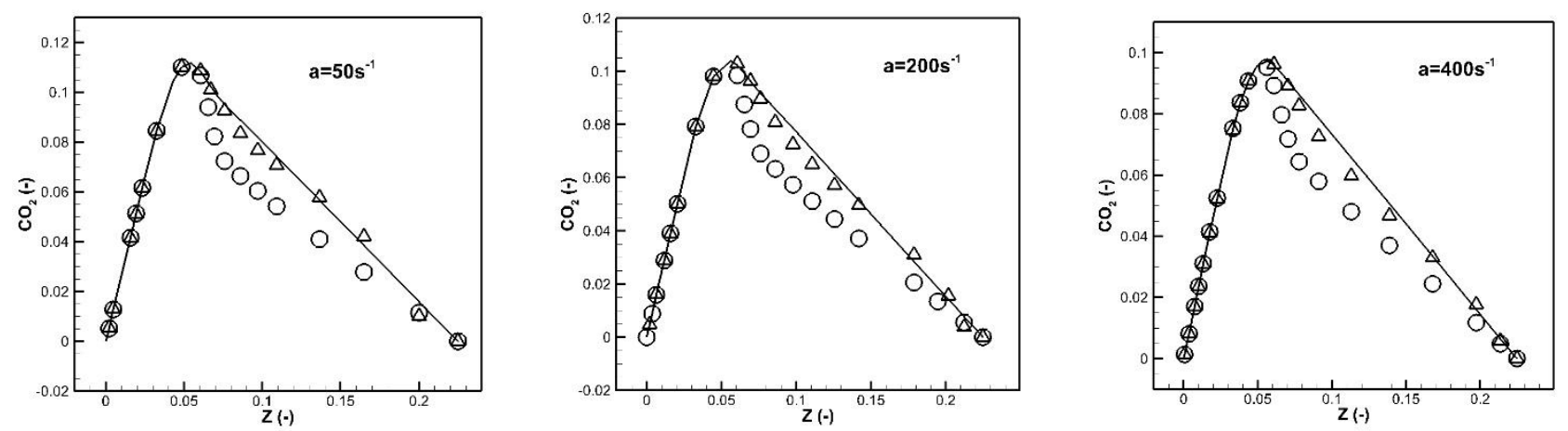

(b)
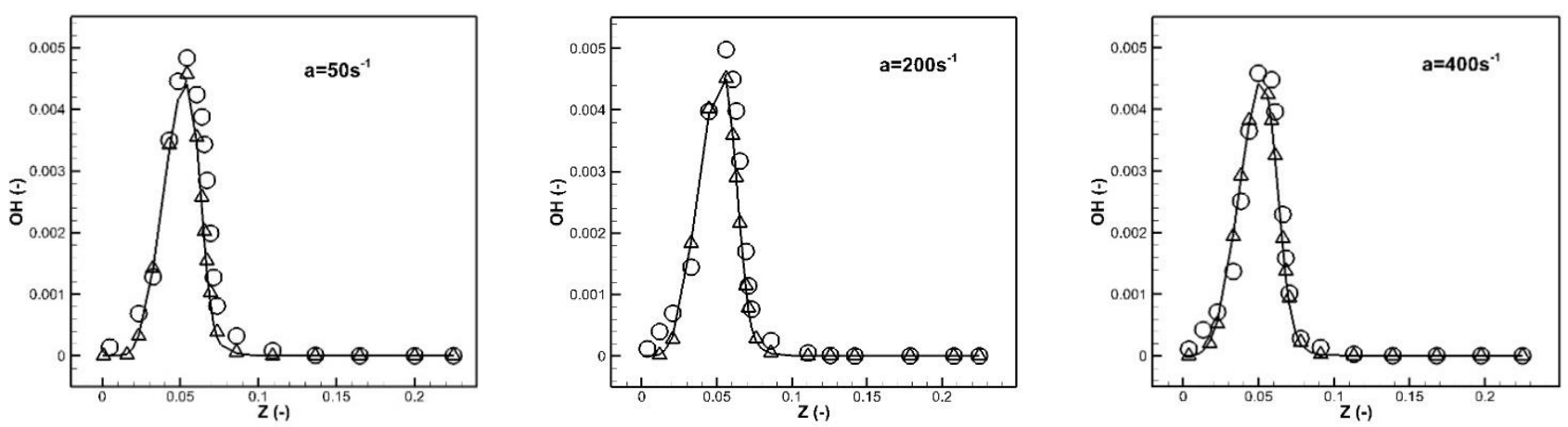

(c) 

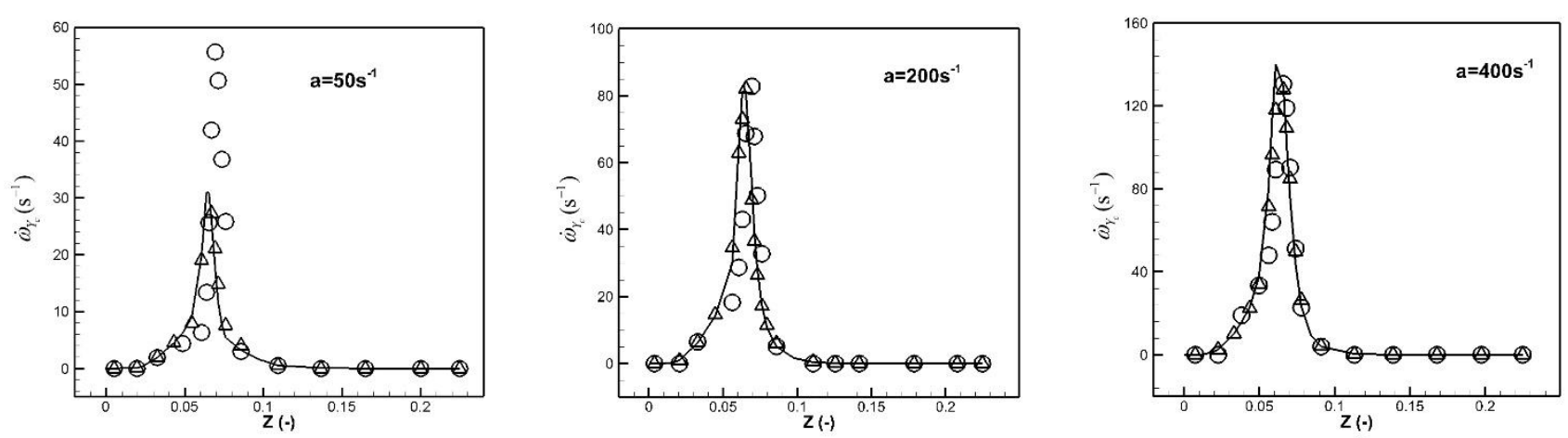

(d)

Fig. 6 Comparisons among different modeling solutions for flames with fuel equivalence ratio $\phi_{F}=5.0$ under different strain rate $a$. (a)Temperature, (b) $Y_{\mathrm{CO}_{2}}$, (c) $Y_{\mathrm{OH}}$, (d) $\dot{\omega}_{Y_{c}}$. Line: detailed chemistry reference solutions. Symbols: Circle, FGM solutions; Delta, Z-c MFM solutions.

Figure 6 shows that temperature is well predicted by both premixed FGM and Z-c MFM approach. However, for mass fractions of $\mathrm{CO}_{2}$ and $\mathrm{OH}$, and $\dot{\omega}_{Y_{c}}$, Z-c MFM approach can still reproduce its distribution accurately while premixed FGM approach failed. Z-c MFM approach can capture local diffusive flame structure for rich partially premixed counter-flow flames against air with fuel equivalence ratio outside of flammable limit.

\subsubsection{Diffusion flames}

For diffusion flames against air with fuel equivalence ratio $\phi_{F}=\infty$, which consist of purely diffusion flame structure, comparisons among different modeling solutions are presented in Fig.7. 

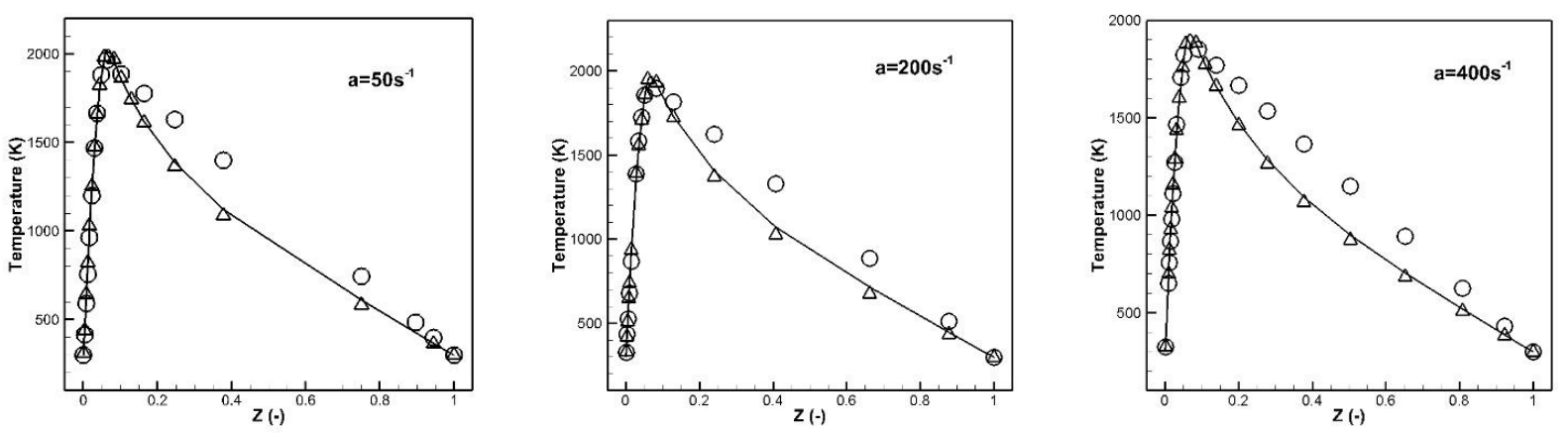

(a)
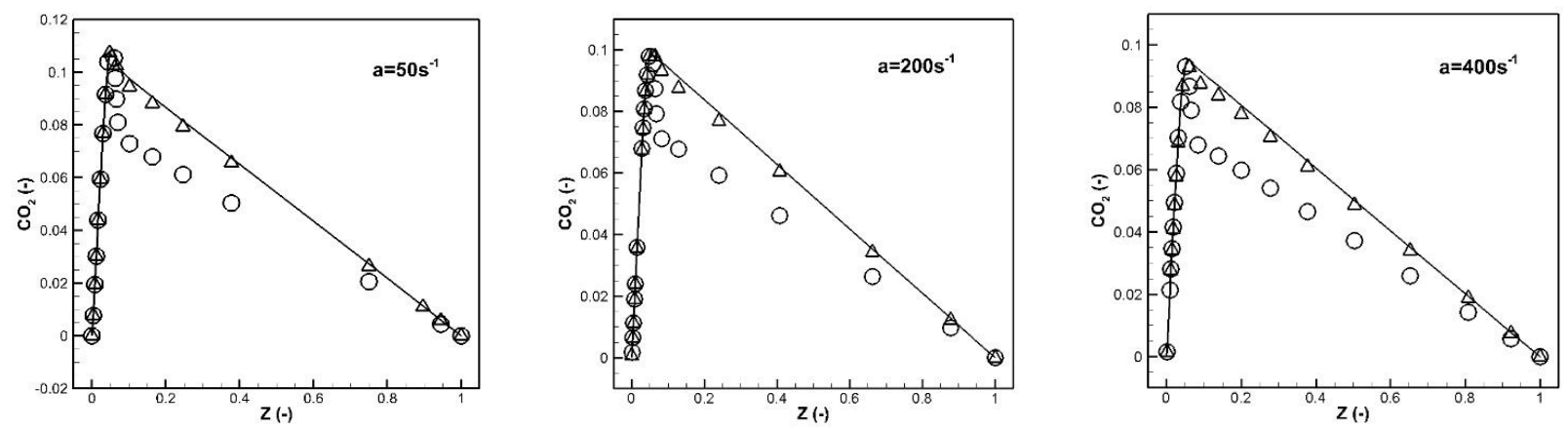

(b)
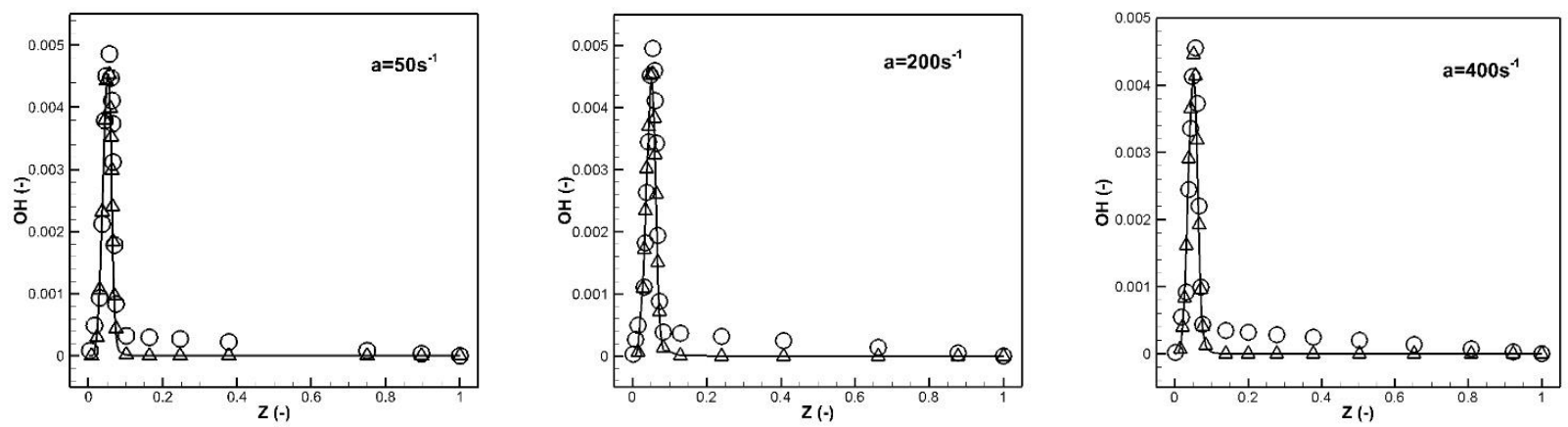

(c)
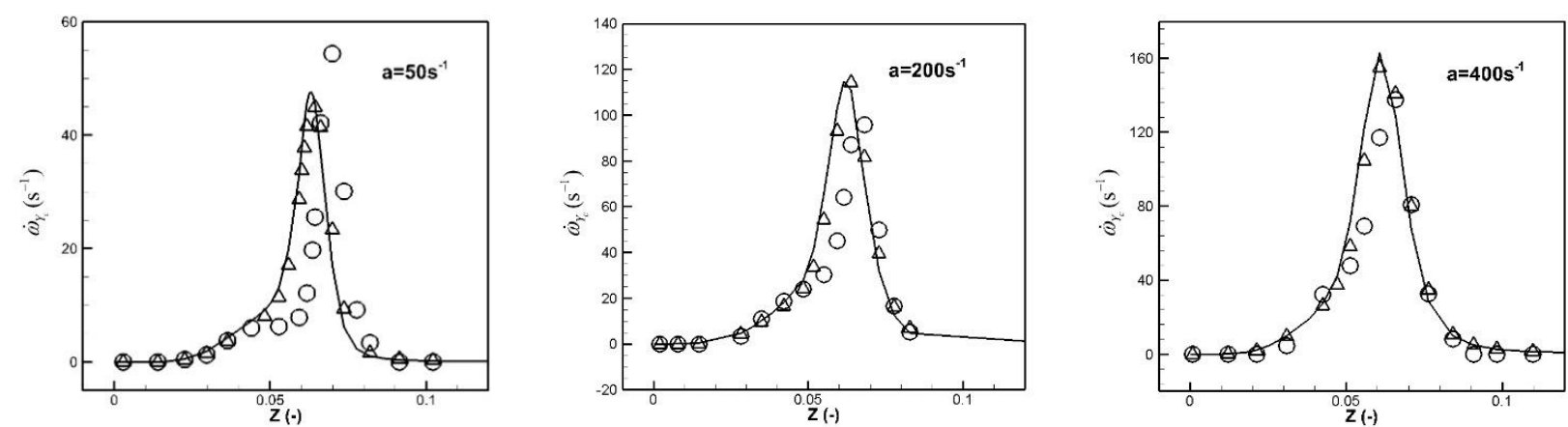

(d)

Fig. 7 Comparisons among different modeling solutions for flames with fuel equivalence ratio $\phi_{F}=\infty$ under different strain rate $a$. (a) Temperature, (b) $Y_{\mathrm{CO}_{2}}$, (c) $Y_{\mathrm{OH}}$, (d) $\dot{\omega}_{Y_{c}}$. Line: detailed chemistry reference solutions. 
Symbols: Circle, FGM solutions; Delta, Z-c MFM solutions

Figure 7 shows that premixed FGM approach failed to reproduce distributions of temperature, mass fractions of $\mathrm{CO}_{2}$ and $O H$, and $\dot{\omega}_{Y_{c}}$, while Z-c MFM approach still proves to be accurate. Z-c MFM approach can reproduce local flame structure in diffusion limit accurately.

\section{Summary}

A new multi-dimensional flamelet generated manifolds approach based on solving multi-dimensional flamelet equations in mixture fraction $\mathrm{Z}$ and normalized flamelet progress variable $c$ has been proposed to capture both non-premixed and premixed flame structure for flame with partially premixed characteristic. Distribution of scalar dissipation rate values for coordinate components as coefficients of the equation set has been modeled bases on local combustion regime analysis for the calculated two-dimensional flamelets. Since a multi-dimensional flamelet is calculated only for each stoichiometric scalar dissipation rate $\chi_{s t}$ value, computational efforts have been reduced by an order of magnitude using this new approach compared to former MFM approach proposed by Nguyen et.al, which has to calculate a multi-dimensional flamelet for each strain rate $a$ and $b_{s}$ value.

Simulation results of counter-flow laminar partially premixed flame shows that solutions obtained through Z-c MFM approach can reproduce both non-premixed and premixed flame structure accurately, while premixed FGM failed. As for rich partially premixed flame with fuel stream $\phi_{F}$ inside the flammability domain, local reaction rate of progress variable $\dot{\omega}_{Y_{c}}$ still cannot be well reproduced by Z-c MFM approach since interactions between diffusive and premixed burning layer are enhanced, which indicates an modified modeling scheme for scalar dissipation rate $\chi_{c}$ has to be proposed in further research to improve the modeling accuracy for Z-c MFM approach.

This work was supported by the National Natural Science Foundation of China (Grant No. 51176178) and the National Natural Science Foundation of China (Grant No. 51376171).

\section{References}

Barlow, R., Karpetis, A., Frank, J. and Chen, J., Scalar profiles and NO formation in laminar opposed-flow partially premixed methane/air flames, Combustion and flame, Vol.127, No.3 (2001), pp.2102-2118.

Barths, H., Peters, N., Brehm, N., Mack, A., Pfitzner, M. and Smiljanovski, V., Simulation of pollutant formation in a gas-turbine combustor using unsteady flamelets, Symposium (International) on Combustion, Vol.27, No.2 (1998), pp.1841-1847.

Brown, P. N., Byrne, G. D. and Hindmarsh, A. C., VODE: A variable-coefficient ODE solver, SIAM journal on scientific and statistical computing, Vol.10, No.5 (1989), pp.1038-1051.

Coelho, P. and Peters, N., Unsteady modelling of a piloted methane/air jet flame based on the Eulerian particle flamelet model, Combustion and Flame, Vol.124, No.3 (2001), pp.444-465.

Darabiha, N., Transient behaviour of laminar counterflow hydrogen-air diffusion flames with complex chemistry, Combustion science and technology, Vol.86, No.(1-6) (1992), pp.163-181.

Dold, J., Flame propagation in a nonuniform mixture: analysis of a slowly varying triple flame, Combustion and Flame, Vol.76, No.1 (1989), pp.71-88.

Domingo, P., Vervisch, L. and Bray, K., Partially premixed flamelets in LES of nonpremixed turbulent combustion. Combustion Theory and Modelling, Vol.6, No.4 (2002), pp.529-551.

Domingo, P., Vervisch, L. and Réveillon, J., DNS analysis of partially premixed combustion in spray and gaseous turbulent flame-bases stabilized in hot air, Combustion and Flame, Vol.140, No.3 (2005), pp.172-195.

Fiorina, B., Baron, R., Gicquel, O., Thevenin, D., Carpentier, S. and Darabiha, N., Modelling non-adiabatic partially premixed flames using flame-prolongation of ILDM, Combust. Theory Model, Vol.7, No.3 (2003), pp.449-470.

Fiorina, B., Gicquel, O., Vervisch, L., Carpentier, S. and Darabiha, N., Approximating the chemical structure of partially premixed and diffusion counterflow flames using FPI flamelet tabulation, Combustion and Flame, Vol.140, No.3 (2005), pp.147-160. 
Fiorina, B., Gicquel, O., Vervisch, L., Carpentier, S. and Darabiha, N., Premixed turbulent combustion modeling using tabulated detailed chemistry and PDF, Proc. Combust. Inst, Vol.30, No.1 (2005), pp.867-874.

Ghosal, S. and Vervisch, L., Theoretical and numerical study of a symmetrical triple flame using the parabolic flame path approximation, Journal of Fluid Mechanics, Vol.415, No.1 (2000), pp.227-260.

Gicquel, O., Darabiha, N. and Thévenin, D., Laminar premixed hydrogen/air counterflow flame simulations using flame prolongation of ILDM with differential diffusion, Proceedings of the Combustion Institute, Vol.28, No.2 (2000), pp.1901-1908.

Kazakov, A. and Frenklach, M., http://www.me.berkeley.edu/drm/.

Knudsen, E. and Pitsch, H., A general flamelet transformation useful for distinguishing between premixed and non-premixed modes of combustion, Combustion and Flame, Vol.156, No.3 (2009), pp.678-696.

Knudsen, E. and Pitsch, H., Capabilities and limitations of multi-regime flamelet combustion models, Combustion and Flame, Vol.159, No.1 (2012), pp.242-264.

Lam, S., Using CSP to understand complex chemical kinetics, Combustion Science and Technology, Vol.89, No.1 (1993), pp.375-404.

Li, S. and Williams, F., NOx formation in two-stage methane-air flames, Combustion and Flame, Vol.118, No.3 (1999), pp.399-414.

Maas, U. and Pope, S. B., Simplifying chemical kinetics: intrinsic low-dimensional manifolds in composition space, Combustion and Flame, Vol.88, No.3 (1992), pp.239-264.

Maas, U. and Pope, S. B., Implementation of simplified chemical kinetics based on intrinsic low-dimensional manifolds, Symposium (International) on Combustion, Vol.24, No.1 (1992), pp.103-112.

Marracino, B. and Lentini, D., Radiation modelling in non-luminous nonpremixed turbulent flames, Combustion science and technology, Vol.128, No.(1-6) (1997), pp.23-48.

Nguyen, P. D., Vervisch, L., Subramanian, V. and Domingo, P., Multidimensional flamelet-generated manifolds for partially premixed combustion, Combustion and Flame, Vol.157, No.1 (2010), pp.43-61.

Oijen, J. V. and Goey, L. D., Modelling of premixed laminar flames using flamelet-generated manifolds, Combustion Science and Technology, Vol.161, No.1 (2000) pp.113-137.

Peters, N., Laminar diffusion flamelet models in non-premixed turbulent combustion, Progress in energy and combustion science, Vol.10, No.3 (1984), pp.319-339.

Peters, N., Proc Combust Inst, Vol.21 (1986), pp.1231-1250.

Peters, N., Turbulent combustion. 2000.

Pierce, C. D. and Moin, P., Progress-variable approach for large-eddy simulation of non-premixed turbulent combustion, Journal of Fluid Mechanics, Vol.504, No.1 (2004), pp.73-97.

Pitsch, H. and Lageneste, L. D., Large-eddy simulation of premixed turbulent combustion using a level-set approach, Proceedings of the Combustion Institute, Vol.29, No.2 (2002), pp.2001-2008.

Pitsch, H., Wan, Y. and Peters, N., Numerical investigation of soot formation and oxidation under diesel engine conditions, SAE Technical Paper, No.952357 (1995).

Pitsch, H., Improved pollutant predictions in large-eddy simulations of turbulent non-premixed combustion by considering scalar dissipation rate fluctuations, Proceedings of the Combustion Institute, Vol.29, No.2 (2002), pp.1971-1978.

Ravikrishna, R. and Laurendeau, N. M., Laser-induced fluorescence measurements and modeling of nitric oxide in counterflow partially premixed flames, Combustion and flame, Vol.122, No.4 (2000), pp.474-482.

Strang, G., On the construction and comparison of difference schemes, SIAM Journal on Numerical Analysis, Vol.5, No.3 (1968), pp.506-517.

Verwer, J. G. and Sommeijer, B. P., An Implicit-Explicit Runge--Kutta--Chebyshev Scheme for Diffusion-Reaction Equations, SIAM Journal on Scientific Computing, Vol.25, No.5 (2004), pp.1824-1835.

See, Y. C. and Ihme, M., Large eddy simulation of a partially-premixed gas turbine model combustor, Proceedings of the Combustion Institute, Vol.35, No.2 (2015), pp.1225-1234. 